2016-09-06

\title{
Optical Wavelength Ratiometric Monitoring System for Data Centre CWDM Applications
}

\author{
Anthony Colohan \\ Technological University Dublin, anthony.colohan@tudublin.ie \\ Thomas Freir \\ Technological University Dublin, thomas.freir@tudublin.ie \\ Derek Finlay \\ Wood Communications, Ireland, derek@woodcomm.ie
}

Follow this and additional works at: https://arrow.tudublin.ie/engscheleart

Part of the Electrical and Electronics Commons

\section{Recommended Citation}

Colohan, A. et al. (2016). Optical Wavelength Ratiometric Monitoring System for Data Centre CWDM Applications. UPEC:51st International Universities Power Engineering Conference (UPEC 2016). ISEC Engenharia, Coimbra, Portugal, 6-9 September. doi:10.21427/D7WG7R

This Conference Paper is brought to you for free and open access by the School of Electrical and Electronic Engineering at ARROW@TU Dublin. It has been accepted for inclusion in Conference papers by an authorized administrator of ARROW@TU Dublin. For more information, please contact arrow.admin@tudublin.ie, aisling.coyne@tudublin.ie, gerard.connolly@tudublin.ie.

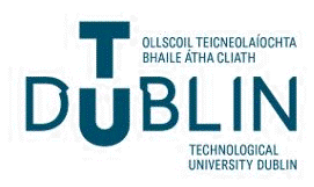




\section{Optical Wavelength Ratiometric Monitoring System for Data Centre CWDM Applications}

\author{
Anthony Colohan \\ Dublin Institute of Technology, \\ Ireland \\ Anthony.Colohan@dit.ie
}

\author{
Thomas Freir \\ Dublin Institute of Technology, \\ Ireland \\ Thomas.Freir@dit.ie
}

\author{
Derek Finlay \\ Wood Communications, \\ Ireland \\ derek@woodcomm.ie
}

\begin{abstract}
-
The development of the internet and the subsequent increase in the quantity of connected devices in recent years has given rise to an explosion in demand of data traffic across telecommunication networks. Converged networks deliver high speed bit rates for the purpose of streaming videos, downloading music files and communicating over the internet; voice over internet protocol (VOIP). Further additional data traffic growth stemming from the upsurge in the amount of 'tweets' and instant messages (IM) created as well as emails written and cloud storage, has led to a demand for the construction of much larger data centres, which have the capacity to store and transmit this increased data. The physical location of these larger data centres is often governed by their proximity to the source of cheap electrical power and favourable climate conditions.

In this paper, the development of an optical fibre monitor is examined. The main attributes of the system will be its ability to monitor both the signal optical power and an individual wavelength within a live coarse wavelength division multiplexing (CWDM) fibre optic installation without causing any disturbance to the link. In response to the prolific growth of larger data centres and the increased demand for CWDM links to service them the onus to develop a low cost, flexible and reliable alternative to the spectrum analyser for the purpose of monitoring has intensified.
\end{abstract}

Index Terms - Optical Networks, Ratio-Metric Monitor, Electrical Services, CWDM Applications, Data Centre.

\section{INTRODUCTION}

Updating of backbone infrastructure to accommodate the required increase in bit rates that data centres demand can be expensive and cumbersome to install, the costs incurred are a result of cable and component prices, payments for installation and for rights of way. Estimated costs associated with the installation of new fibre optical cables are expensive and careful design is essential to keep costs to a minimum [1]. A more cost effective alternative is the process of multiplexing wavelengths when network expansion and increased capacity are required. The technique known as WDM (wave-division multiplexing) combines and transmits multiple wavelengths in the same fibre. Instead of adding multiple fibres per channel each channel of information transmitted through the fibre is allocated an individual wavelength and the combined wavelengths within the signal are then separated on arrival at the receiver.

The benefit of adopting a multiplexed optical fibre system over a new fibre installation makes it a far more desirable choice when increased demand is required. Analysts estimate growth in traffic coupled with observed trends in commercial practice will also result in a requirement for interfaces to the core network to migrate from the current $10 \mathrm{~Gb} / \mathrm{s}$ and $40 \mathrm{~Gb} / \mathrm{s}$ to $100 \mathrm{~Gb} / \mathrm{s}$ in the next few years and to $1 \mathrm{~Tb} / \mathrm{s}$ by 2020 [2]. Data centre designers and operators are extremely cognizant of the cost, power consumption and space afforded to optical fibre installations. The current ITU-T G.695 recommendation provides a framework to support the growth and needs of next generation data centres. The recommendation classifies the parameters that cater for physical point -to- point and ring CWDM system applications that are available for $2 \mathrm{~km}$ short haul CWDM links used to connect data centre high speed communication racks together and for metro links of up to $40 \mathrm{~km}$ providing the connections between data centres. Data centre designers in this regard are now looking to deploy CWDM, a cost effective solution that can deliver bit rates of $2.5 \mathrm{Gbits} / \mathrm{s}$ and $10 \mathrm{Gbits} / \mathrm{s}$ over $4,8,12$ and 16 channels. [3].

A key difference between dense wavelength division multiplexing (DWDM) and CWDM is the wavelength channel spacing; with DWDM having channel spacing's as low as $0.2 \mathrm{~nm}$. CWDM's large channel spacing of $20 \mathrm{~nm}$ allows the use of low cost uncooled lasers, multiplexers and de-multiplexers with much lower tolerances. DWDM systems use complex temperature control techniques, using a peltier cooler, to keep the temperature of the DWDM laser source constant as the wavelength of a distributed feedback laser (DFB) source drifts with temperature typically by $0.1 \mathrm{~nm}$ per degree Celsius (4). CWDM sources on the other hand are not temperature controlled which leads to a much simpler design and hence a significant reduction in cost. The central wavelength of each CWDM source is allowed a tolerance of $\pm 6.5 \mathrm{~nm}$ to account for temperature drift and a manufacturing tolerance. Due to the uncooled nature of CWDM systems, detection of temperature induced wavelength drift of 
individual wavelengths and laser tolerance may require long term monitoring of the fibre installation, possibly over weeks and months. This process often proves challenging to implement as the financial costs associated with leaving expensive test equipment connected for long periods to detect wavelength drift can be burdensome for a service provider.

This paper investigates the potential to develop a cost effective system which can measure the level of optical power and monitor a particular wavelength within a live CWDM system prior to it being multiplexed (or after it has been de-multiplexed) without the necessity to disturb or turn off the existing fibre installation. A key component of the system will be a wavelength dependant filter which can be employed across the whole CWDM wavelength range.

\section{WAVELENGTH MONITORING RATIONALE}

The two forms of wavelength multiplexing fibre optical signals are DWDM and CWDM; both techniques in principle are essentially the same the only differences being the size of the channel spacing between wavelengths and the range of the optical spectrum that they cover. The CWDM standard ITU (International Telecommunication Union) recommendation G.694.2 classifies a wavelength range that stretches between $1271 \mathrm{~nm}$ and $1611 \mathrm{~nm}$ [5]. The CWDM application specifies 18 wavelengths with $20 \mathrm{~nm}$ spacing between channels with systems containing $4,8,12$ or 16 channels. On long haul commercial optical fibre installations above $40 \mathrm{~km}$ DWDM is the preferred option, the channels are much more tightly packed across the C \& L optical spectral bands where the optical signals exposure to attenuation and dispersion is far less than is found in other bands and optical amplification using optical amplifiers is also possible. Channel spacing can be as low as $0.2 \mathrm{~nm}$ providing the potential to deliver $80+$ individual channels of information with speeds of $10 \mathrm{Gbits} / \mathrm{sec}$ and beyond per channel [6].

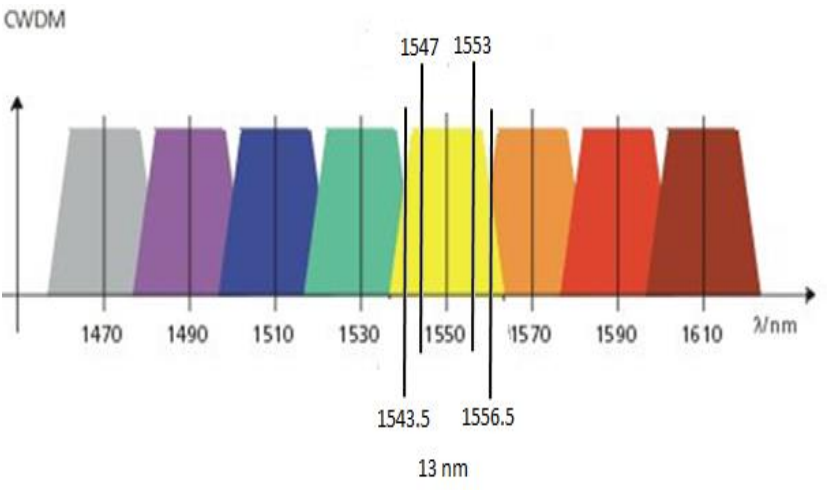

Fig 1. 8 channel CWDM spectrum with the $1550 \mathrm{~nm}$ source wavelength variation due to manufacturers $(1547-1553 \mathrm{~nm})$ and temperature (1543.5 to $556.5 \mathrm{~nm}$ ) tolerance labelled.

The nature of the CWDM fibre topology consists of a rack containing 4 input ports connected to 4 individual lasers. The 4 wavelengths emitted by the individual lasers have a successive separation range of $20 \mathrm{~nm}$, these 4 individual optical signals are multiplexed within the rack and the resulting CWDM optical signal is connected to the fibre via the interconnect output port and mixed with another CDWM signal if more channel capacity is required. The operating characteristics of an uncooled distributed-feedback (DFB) laser exhibits an operating temperatures range that stretches between $0-70^{\circ} \mathrm{C}$. This wide temperature variation can cause the laser's unique wavelength to drift as much as \pm 3.5 $\mathrm{nm}$.

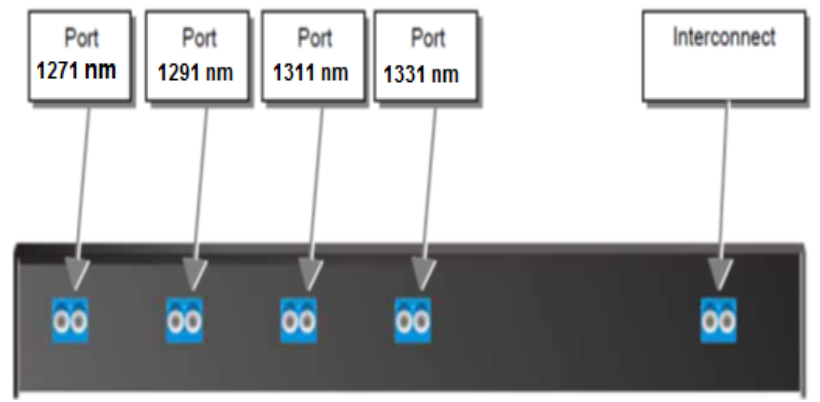

Fig. 2. CWDM Optical Multiplexer 4 Way Rack

The wavelength temperature drift, coupled with the laser manufacturer's wavelength transmitter tolerance of up to \pm 3 $\mathrm{nm}$ [7] can produce an overall wavelength variation of approximately $\pm 6.5 \mathrm{~nm}$. The resulting wavelength drift towards an adjacent channel can have a detrimental effect on the transmission of information within the CWDM optical link. If an individual channel drifts beyond $\pm 6.5 \mathrm{~nm}$ from its central wavelength position it enters the stopband of the optical filter within the multiplexer and de-multiplexer, this results in severe attenuation. The implementation of the optical monitor connected to each individual input or output channel could detect a wavelength drifting beyond its $6.5 \mathrm{~nm}$ tolerance into another channels spacing, once identified the necessary action can be taken to restore channel service and resolve any cross channel interference discovered.

\section{SPECTRUM ANALYSER VS OPTICAL WAVELENGTH MONITOR}

As the name suggests an optical spectrum analyser provides analysis of optical power as a function of wavelength. These instruments offer accurate high resolution testing on both laser and LED (Light Emitting Diode) light sources, results captured provide the spectral response and power $(\mathrm{dB})$ distribution of an optical signal across multiple wavelengths. Additionally, the device can also provide in depth analysis of the optical power transmission characteristics of fibre optical components such as couplers.

However, this instrument is not suitable for long term onsite monitoring. The light source entering the instrument is spatially dispersed by a rotating diffraction grating which 
slows down the speed of measurement and this also raises concerns regarding the presence of vibration distorting the instrument readings. Furthermore, the nature of the mechanical rotating components imposes restrictions on locations where the instrument can be installed for the purpose of testing. A spectrum analyser should only be used in an environment which satisfies the following criteria:

- Ambient temperature: $10^{\circ} \mathrm{C}$ to $40^{\circ} \mathrm{C}$ (operating temperature)

- Relative humidity: $85 \%$ or less (without condensation)

- An area free from corrosive gas

- An area away from direct sunlight

- A dust free area

- An area free from vibrations

- A low electrical noise area

The high financial cost associated with the spectrum analyser deems it in some instances not a viable economical option for monitoring of wavelength drift over long periods. Additional financial costs incurred over its lifespan include yearly calibration and the maintenance of the instrument's main parts, for example the replacement of the unit power supply, LCD (Liquid Crystal Display), LCD back light, fan motor and electrolytic capacitor.

The proposed optical monitor using an optical wavelength ratiometric system is a feasible low cost alternative which does not suffer the same environmental restraints experienced by the spectrum analyser. This is largely due to the fact that the device has no mechanical moving parts. Although the instrument may not achieve the same high level of accuracy as the spectrum analyser it does exhibit sufficient potential instrument accuracy for the purpose of long term monitoring of individual CWDM optical signals on a cost effective basis.

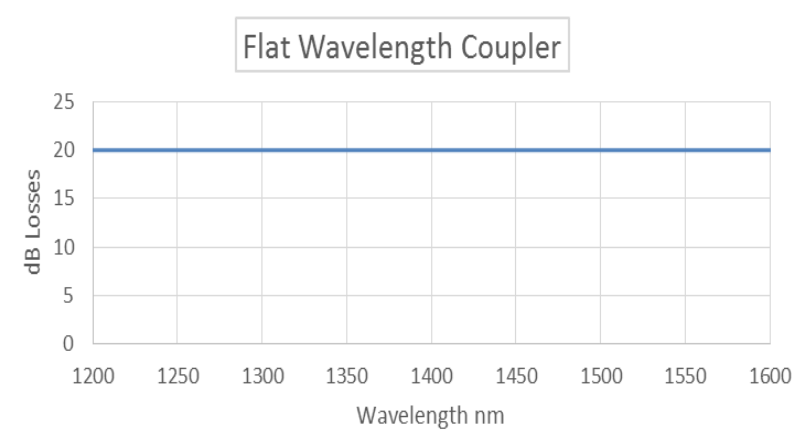

Fig. 3. Wavelength Flat Coupler

The system is also reasonably fast in providing results; its measurement is only limited by the response time of the filter and the speed of the analogue to digital conversion. On testing a live fibre optical installation the optical ratiometric system's ability to not cause any further disturbance relies on the 99/1 optical taps typically supplied at both the transmitter and receiver ends of CWDM systems. These tap couplers are positioned at the point prior to multiplexing and after demultiplexing of the wavelengths occur.

The preinstalled 99/1 tap couplers are required to be wavelength flat couplers. The coupler taps $1 \%$ of the live optical signal and feeds it into the ratiometric monitor whilst the main portion of the live optical signal $(99 \%)$ is delivered to the network as normal. The optical power attenuation characteristics of this type of coupler ensure that no erroneous values are introduced if wavelength drift occurs on the optical signal. One drawback of the system is that unlike its counterpart the spectrum analyser the optical wavelength ratiometric system is a single channel device which only has the capability to analysis one wavelength at a time.

\section{RATIO-METRIC POWER MEASUREMENT TECHNIQUE}

Optical couplers are used to split, combine and route signals within fibre optic systems and their functions form an integral part of wavelength division multiplexing/demultiplexing of telecommunication networks. Optical couplers are designed to be either wavelength independent with a flat wavelength response or wavelength dependant.

Wavelength dependant couplers are used commercially to both combine and separate optical signals with different wavelengths, the coupling coefficient of these devices is dependent on the wavelengths contained within the optical signal.

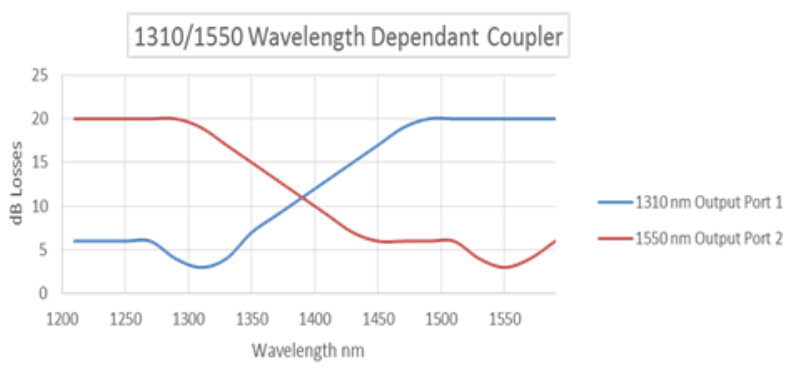

Fig. 4. Wavelength Dependant Coupler

This selective process permits the passage of a specific wavelength through one of the output ports whilst restricting the optical power of all other wavelengths through that same output port. There are a number of different wavelength dependant coupler combinations commercially available e.g. 980/1550, 1310/1550. The optical power response of a wavelength dependant coupler is depicted in figure 4 above; the output behaviour resembles the characteristics of an optical filter.

As the multiplexed optical signal is passed through the coupler a unique $\mathrm{dB}$ power loss is attenuated across each wavelength. The optical power loss affiliated to each individual wavelength can be used to identify the presence of that wavelength within the multiplexed optical signal once the optical power/wavelength characteristics of the coupler are captured on a spectrum analyser using $0.01 \mathrm{~dB}$ increments. The case for considering an optical coupler as a 
filter is further bolstered by the fact that the device has large discrimination properties due to the potential $20 \mathrm{~dB}$ loss across its full wavelength spectrum. The filters' large discrimination due to the $0 \mathrm{~dB}$ to $20 \mathrm{~dB}$ loss across the wavelength range provides a high resolution, considering a typical optical power meter can measure to a $0.01 \mathrm{~dB}$ resolution $20 \mathrm{~dB} \div 0.01 \mathrm{~dB}=2000$ digital units $(d u)$ spread over the wavelength range of an commercial off the shelf coupler $1271 \mathrm{~nm}$ and $1551 \mathrm{~nm}$ the device provides a filter accuracy of $[(1551-1271) \div 2000 \mathrm{du}]=0.14 \mathrm{~nm}$. The ratiometric optical wavelength monitor can detect source wavelength changes of $0.14 \mathrm{~nm}$.

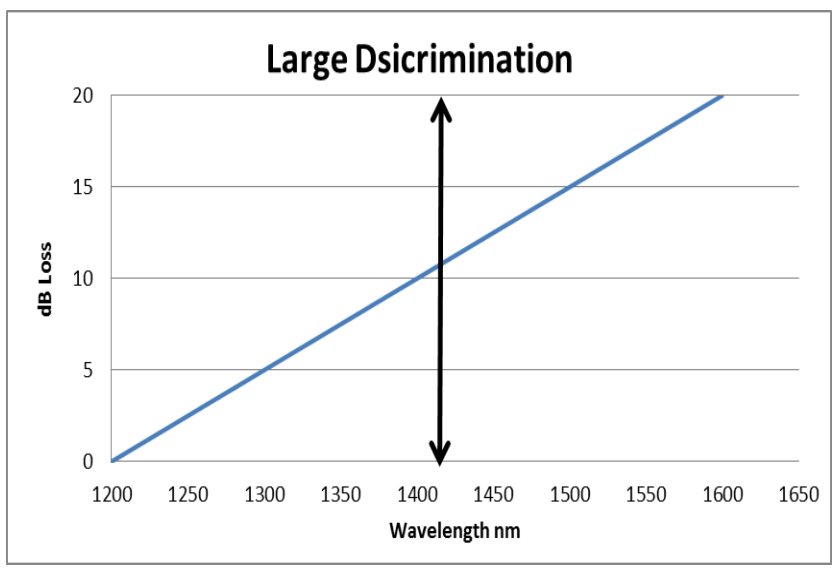

Fig. 5. Filter Wavelength Discrimination

Rather than just merely recording the optical power lost at the filter the Ratio-Metric technique compares the output optical power values recorded at both ports of the coupler. This technique ensures that the power loss ratio between the two output ports at each specific wavelength will always be the same irrespective of any power loss variance occurring on the optical signal entering the input side of the device. The optical ratiometric system in figure 6 below illustrates how the optical power ratio of $20 \mathrm{~dB}$ is maintained between the two output ports regardless of any deviation in the level of optical power entering the system via the optical transmitter.
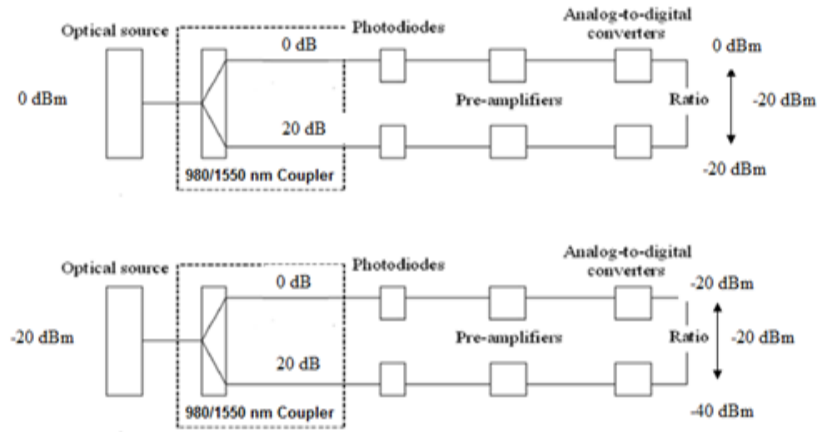

Fig. 6 Basic Ratio-Metric Wavelength Detection System at a known wavelength with the coupler acting as an optical wavelength filter
The variation in optical input power could be a result of optical add-drop multiplexers (OADM) being added or removed for the purpose of multiplexing and routing channels into or out of an active optical fibre installation. Just like the optical fibre couplers these devices have their own unique optical fibre power characteristics that will change the optical power level when added or removed from an active optical fibre installation.

\section{SYSTEM IMPLEMENTATION}

The optical signals from the coupler's output ports are passed through two individual photodiodes which change the optical signals into electrical currents, the attributed sensitivity of each photodiode defines the ratio of output electrical current to optical input power, the responsivity of the photodiode is typically quantified in amperes/watt or sometimes volts/watt. After the optical-to-electrical current conversion has taken place both electrical currents are amplified and converted to a voltage which will then provide a proportional representation of the optical power ratio corresponding to the measured wavelength. A photodiode comprising of a large surface area is necessary to capture all the light from a fibre when optical power monitoring is required. A photodiode's characteristic is defined by its responsivity $\mathcal{R}$ (1), where the average output current generated by the photodiode is a result of the incident optical input power $P_{\text {in }}$ watts.

$$
\mathcal{R}=\frac{I_{p}}{P_{\text {in }}} \mathrm{A} / \mathrm{W}
$$

The InGaAs G8194 photodiode exhibits a suitable spectral response for the optical wavelength Ratio-Metric System; the large surface area of the InGaAs G8194 photodiode enables it to capture all the optical power from the fibre. However, the photodiodes large surface area introduces a large amount of parasitic capacitance and this will have a bearing on the conversion time of the optical signal into an electrical signal. This can be largely ignored because the speed at which the system processes the results is sufficiently adequate.

The two measured converted output voltages provide a ratio (2) that can be correlated with a predefined voltage to wavelength lookup table for the purpose of identifying the presence or absence of a specific wavelength within the optical signal.

$$
V_{\text {out }}=\frac{V_{1}}{V_{2}}
$$

Although this method of optical power measurement can successfully measure the power ratio between two optical signals the dynamic range of this method is questionable when the device is required to be interchanged between the 
signals transmitter and receiver. The difference in optical power between the transmitter and receiver can be as large as $40 \mathrm{~dB}$ and the output current from the photodiode can swing from $\mu A$ to $\mathrm{mA}$. The resulting output voltage from the converter can vary from Volts to $\mathrm{kV}$, this variation is obviously impractical and a form of range switching is required to adjust the current - to - voltage feedback resistor $R_{f}$ accordingly to control the amplification process of the converter. However, this method is too cumbersome to achieve as the switching of the resistor must be regulated automatically to match the change in level of optical input power entering the system.

The dynamic range problem can be overcome by employing two logarithmic current to voltage convertors that can successfully convert the two photodiode currents to voltages. The output voltage from these devices is a linear function of the logarithm of the input current delivered from the photodiode. There are a number of logarithmic current to voltage convertors available but the ThermOptics ${ }^{\mathrm{TM}}$ DN135 is specifically designed for use in optical fibre power monitors incorporating photodiodes

The ThermOptics ${ }^{\mathrm{TM}}$ DN135 lookup table illustrated below indicates the range of converted input current to output voltage values that it can accommodate. It also identifies a corresponding optical power level $(\mathrm{dBm})$ which is used to ascertain the unique wavelength form the results captured from the spectrum analyser.

TABLE I.

ThermOptics ${ }^{\text {TM }}$ DN135 Lookup Table

\begin{tabular}{|rc|c|c|}
\hline \multicolumn{3}{|c|}{ INPUT } & OUTPUT \\
POWER & VOLTAGE \\
\hline Watts & $\mathrm{dBm}$ & Volts \\
\hline 1 & $\mathrm{~mW}$ & 0 & 4.000 \\
100 & $\mu \mathrm{W}$ & -10 & 3.500 \\
10 & $\mu \mathrm{W}$ & -20 & 3.000 \\
1 & $\mu \mathrm{W}$ & -30 & 2.500 \\
100 & $\mathrm{nW}$ & -40 & 2.000 \\
10 & $\mathrm{nW}$ & -50 & 1.500 \\
1 & $\mathrm{nW}$ & -60 & 1.000 \\
100 & $\mathrm{pW}$ & -70 & 0.500 \\
\end{tabular}

\begin{tabular}{|c|c|}
\hline $\begin{array}{c}\text { INPUT } \\
\text { CURRENT }\end{array}$ & $\begin{array}{l}\text { OUTPUT } \\
\text { VOLTAGE }\end{array}$ \\
\hline Amperes & Volts \\
\hline $1 \mathrm{~mA}$ & 4.000 \\
\hline $100 \mu \mathrm{A}$ & 3.500 \\
\hline $10 \mu \mathrm{A}$ & 3.000 \\
\hline $1 \mu \mathrm{A}$ & 2.500 \\
\hline $100 \mathrm{nA}$ & 2.000 \\
\hline$n A$ & 1.500 \\
\hline $1 \mathrm{nA}$ & 1.000 \\
\hline $100 \mathrm{pA}$ & 0.500 \\
\hline
\end{tabular}

The output voltage is a linear function of the logarithm of input current, hereafter the anticipated ratio in $\mathrm{dBm}$ can be affiliated to the difference between the output voltages $V_{1} \& V_{2}(3)$.

$$
\begin{gathered}
P_{d B m 1}=20\left(V_{01}-4\right) d B m \\
P_{d B m 2}=20\left(V_{02}-4\right) d B m \\
P_{d B m 1}-P_{d B m 2}=20\left(V_{01}-4\right)-20\left(V_{02}-4\right) d B m \\
P_{d B m 1}-P_{d B m 2}=20 V_{01}-80-20 V_{02}+80 d B m \\
P_{d B m 1}-P_{d B m 2}=20 V_{01}-20 V_{02} \\
P_{d B m 1}-P_{d B m 2}=20\left(V_{01}-V_{02}\right)
\end{gathered}
$$

\section{EXPERIMENTAL RESULTS AND DISCUSSION}

For this proof of concept device the filter selected is an off the shelf 3 port $980 / 1550 \mathrm{~nm}$ optical coupler with two output ports that each demonstrate a clearly defined spectral response; both spectral responses ideally act in opposition to each other as depicted in Fig. 7 below. The more linear and converse the two spectral responses are the greater the enhancement of the resolution of the measurement device. An optical power ratio between the two output ports which is a function of wavelength can be attributed to each specific wavelength.

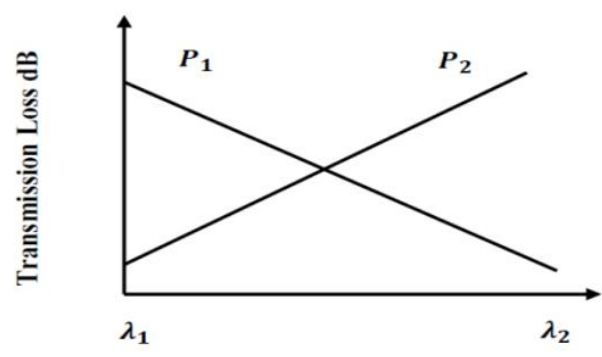

Fig. 7. Ideal Spectral Response from 980/1550 nm Coupler

Initially a reference power loss in $\mathrm{dB}$ for the light source is identified with the use of a spectrum analyser (ADVANTEST 8384 ) to capture and record the source optical power loss across each wavelength. The light source is then reconnected to the input side of the coupler and the spectrum analyser captures the optical $\mathrm{dB}$ power loss across all wavelengths on each of the filter's two output ports.

The initial reference optical power loss which takes account of the inherent light source power loss at each wavelength is subtracted from the optical power loss captured at the corresponding wavelength on each of the filter's output ports. These two sets of results which now reflect the true optical power loss of each output port are subtracted from one another to give the ratio-metric optical power measurement at each wavelength. Output Port 2 permits the passage of 980 $\mathrm{nm}$ wavelength signals with the minimum of $\mathrm{dB}$ loss whilst attenuating the signal strength of all other wavelengths. Port 3 on the contrary permits the passage of $1550 \mathrm{~nm}$ wavelength signals with the minimum of $\mathrm{dB}$ loss whilst attenuating all other signals below this wavelength.

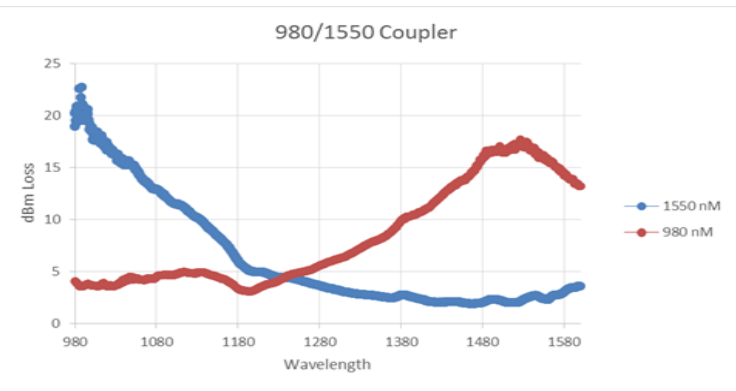

Fig. 8. 980/1550 nm Coupler Spectral Response. 
The characteristic performance of the $980 \mathrm{~nm} / 1550 \mathrm{~nm} 3$ port $\mathrm{T}$ coupler captured on the spectrum analyser (ADVANTEST 8384) is illustrated in Fig. 8 above.

The two ThermOptics ${ }^{\mathrm{TM}}$ DN135 current to voltage convertors connected to the InGaAs (Indium Gallium Arsenide) photodiodes were calibrated by passing a $1500 \mathrm{~nm}$ wavelength at a power rating of $-10 \mathrm{dBm}$ through the input side of the photodiodes via a tuneable laser. As the tuneable laser only had the capacity to deliver $\pm 7 \mathrm{dBm}$ the fibre optic cable was turned a number of times to form a helix, this introduced a further $-3 \mathrm{dBm}$ in bend losses. The variable resistor on each of the ThermOptics ${ }^{\mathrm{TM}}$ DN135 was adjusted until the desired 3.5 volts was recorded on output pin 9 on each of the current to voltage convertors.

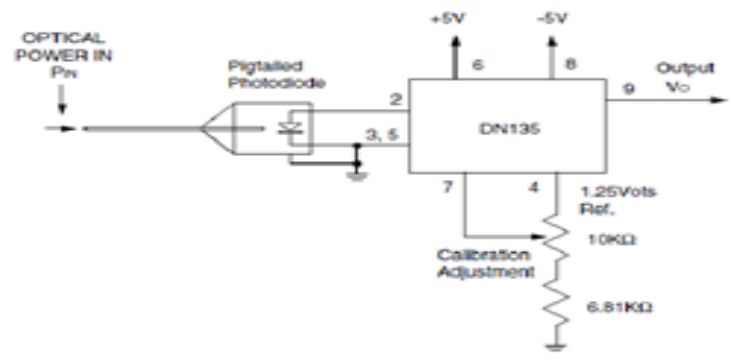

Figure 9: ThermOptics ${ }^{\mathrm{TM}}$ DN135 Schematic Diagram

The change in output voltage on pin 9 is a linear function of the logarithm of input current from the InGaAs G8194 photodiode; therefore the anticipated ratio in $\mathrm{dBm}$ which identifies the optical wavelength can now be associated to the difference between the two output voltages. This electrical voltage change is quantified in digital units with the aid of an Arduino Uno microcontroller and an Arduino code is written to provide a visual display of the wavelength represented on each of the 4 channels.

The tuneable laser was initially set to a power level of $-10 \mathrm{dBm}$ at a wavelength of $1500 \mathrm{~nm}$ and connected to the system. The laser's wavelength was increased in steps of 2 $\mathrm{nm}$ and the corresponding du values were recorded from the display. These recorded du values illustrated in the table II below are correlated with the corresponding $\Delta V=V_{1}-V_{2}$ values captured on output pin 9 of both current to voltage convertors every time the optical signal wavelength is adjusted by $2 \mathrm{~nm}$. Using t $\Delta V=V_{1}-V_{2}$ the corresponding wavelength can be identified from the ThermOptics ${ }^{\mathrm{TM}}$ DN135 lookup table and the resulting wavelength can be matched to the appropriate du value in the Arduino code to provide a live graphical visual display of the wavelength within the live optical fibre channel.

TABLE II.

Arduino Microcontroller Wavelength Representation

\begin{tabular}{|c|c|}
\hline Wavelength & Digital Units \\
\hline $1500 \mathrm{~nm}$ & 268 \\
\hline $1502 \mathrm{~nm}$ & 272 \\
\hline $1504 \mathrm{~nm}$ & 275 \\
\hline $1506 \mathrm{~nm}$ & 279 \\
\hline $1508 \mathrm{~nm}$ & 284 \\
\hline $1510 \mathrm{~nm}$ & 289 \\
\hline $1512 \mathrm{~nm}$ & 295 \\
\hline $1514 \mathrm{~nm}$ & 302 \\
\hline $1516 \mathrm{~nm}$ & 308 \\
\hline $1518 \mathrm{~nm}$ & 313 \\
\hline $1520 \mathrm{~nm}$ & 318 \\
\hline $1522 \mathrm{~nm}$ & 322 \\
\hline $1524 \mathrm{~nm}$ & 327 \\
\hline $1526 \mathrm{~nm}$ & 331 \\
\hline
\end{tabular}

Initial testing of the system revealed that there was a significant amount of electrical noise contained in both of the two output voltages $V_{1} \& V_{2}$. Possible causes of this problem include photodiode shot noise, transimpedence amplifier noise and the quantization noise of the analog-to digital converter (ADC) [8]. The electrical noise stems from the measurement of low levels of optical power, the photodiode output current levels that feed into the DN135 can be in some instances in the region of Nano amperes. It was necessary to build a $10 \mathrm{~Hz}$ low pass filter to overcome this problem of electric noise and also to de-couple the DN135 + $5 \&$ - 5 volt dc supply from ground by connecting a $0.1 \mu F$ capacitor across each rail and ground.

The experimental results obtained were limited by the tuneable laser's ability to only move the source wavelength by $2 \mathrm{~nm}$ increments. However, the calculations demonstrated that the system could actually detect a wavelength shift of $0.14 \mathrm{~nm}$. The systems overall accuracy can be further enhanced with the aid of software that can implement other interpolation techniques.

\section{CONCLUSION}

The development of the optical wavelength ratiometric measurement system and the empirical evidence gathered highlighted the capability of the system to monitor a CWDM optical fibre signal for wavelength shifting detection with an initial resolution of $2 \mathrm{~nm}$. A refined system with an improved resolution approaching $0.2 \mathrm{~nm}$ affords the potential to adequately detect optical fibre wavelength drift problems experienced as a result of the combination of temperature drift and laser tolerance. However, there is room for improving the system's precision, measurement consistency and response time. Furthermore, the system could be enhanced with the design of a purpose built optical coupler (filter) which demonstrates the appropriate wavelength attenuation properties to provide higher levels of accuracy and the ability to measure across the complete CWDM optical spectrum range $(1271 \mathrm{~nm}-1551 \mathrm{~nm})$. The issue of electrical noise in the electronic circuit due to the low levels of optical power being monitored can be addressed by building a proper PCB with a sufficient ground plane.

This cost effective and flexible system which has no moving parts offers optical fibre service providers the ability to connect and measure the existence of a particular wavelength and the level of associated optical power without provoking any further disturbance to a live CWDM fibre optical signal. 


\section{REFERENCES}

[1] U.S. Department of Transportation (2014) "'Equipment Costs for Roadside Telecommunications (RS-TC)", [Online]. Available: http://www.itscosts.its.dot.gov/its/benecost.nsf/DisplayRUCByUnitCos tElementUnadjusted?ReadForm\&UnitCostElement=Fiber+Optic+Cabl e+Installation+\&Subsystem=Roadside+Telecommunications +

[2] Robert W. Tkach, 'Scaling Optical Communications for the Next Decade and Beyond", Bell Labs Technical Journal 14(4), 3-10 2010

[3] ITU-T TELECOMMUNICATION STANDARDIZATION SECTOR OF ITU" Optical interfaces for coarse wavelength division multiplexing applications" G.965 (01/2015)

[4] Hans Joerg Thiele, Coarse wavelength Division Multiplexing Technologies and Applications, Boca Raton Florida: Taylor \& Francis Inc, 2007,p.63.

[5] ITU-T TELECOMMUNICATION STANDARDIZATION SECTOR OF ITU 'Spectral grids for WDM applications: CWDM wavelength grid"'G.964.2 (12/2003)

[6] Vikram Shete, Yiyong Zha, Lei Wang, Yuhua Chent " Cost Analysis of DWDM Multi-Mode Switching Router!' International Conference on High Performance Switching and Routing 2010

[7] MARCUS NEBELING, Fiber Network Engineering " CWDM: lower cost for more capacity in the short-haul " $3^{\text {rd }}$ July 2003.[Online]. Availablehttp://www.cubeoptics.com/uploads/tx_cuboproducts/CUBO_ WP_CWDM_Lower_cost_in_the_short_01.pdf

[8] Ginu Rajan, Yuliya Semenova, Thomas Freir, Pengfei Wang, and Gerald Farrell "Modelling and Analysis of the Effect of Noise on an Edge Based Ratiometric Wavelength Measurement System", JOURNAL OF LIGHTWAVE TECHNOLOGY, VOL. 26, NO. 20, OCTOBER 15, 2008 